\title{
NEURODEVELOPMENTAL AND RESPIRATORY OUTCOMES AT 2 YEARS OF AGE IN PRETERM INFANTS TREATED WITH INHALED NITRIC OXIDE: THE EUNO TRIAL FOLLOW UP
}

\author{
X. Durrmeyer ${ }^{1}$, H. Hummler ${ }^{2}$, M. Sanchez-Luna ${ }^{3}$, V. Carnielli ${ }^{4}$, D. Field ${ }^{5}$, A. Greenough ${ }^{6}$, B. Van \\ Overmeire $^{7}$, B. Jonsson ${ }^{8}$, M. Hallman ${ }^{9}$, J. Baldassarre ${ }^{10}$, N. Marlow ${ }^{11}$, J.-C. Mercier ${ }^{12}$, EUNO Study Group \\ ${ }^{1}$ Réanimation Néonatale, Centre Hospitalier Intercommunal de Créteil, Créteil, France, ${ }^{2}$ Neonatology, \\ Children's Hospital, University of Ulm, Ulm, Germany, ${ }^{3}$ Neonatology, Hospital Universitario Gregorio \\ Marañon, Madrid, Spain, ${ }^{4}$ Neonatology, Polytechnic University of Marche, Mother and Child Institute \\ Ospedale Salesi, Ancona, Italy, ${ }^{5}$ Health Science, University of Leicester, Leicester, ${ }^{6}$ Division of Asthma, \\ Allergy and Lung Biology, Kings College, London, UK, ${ }^{7}$ Neonatology, Erasmus Hospital, Brussels, Belgium, \\ ${ }^{8}$ Neonatology, Karolinska Institute, Stockholm, Sweden, ${ }^{9}$ Neonatology, University Hospital, Oulu, Finland, \\ ${ }^{10}$ Clinical Development, Ikaria, Clinton, NJ, USA, ${ }^{11}$ Institute for Women's Health, University College \\ London, London, UK, ${ }^{12}$ Department of Paediatric Emergency Medicine, Hôpital Robert Debré, Paris, \\ France
}

Background and aims: The EUNO trial of inhaled nitric oxide (iNO) for the prevention of BPD showed similar frequencies for the primary outcome (survival without BPD at 36 weeks postmenstrual age (PMA): iNO 65.3\%; placebo 65.5\%). In this paper, we present post-discharge outcomes including survival free of neurodevelopmental impairment (NDI) and respiratory outcome at 2 years corrected age.

Methods: Babies $<29$ weeks of gestation with moderate respiratory failure in the first 24 hours after birth were randomly allocated iNO $5 \mathrm{ppm}$ for 7 to 21 days or placebo gas. All babies were followed up at 1 and 2 years of corrected age. Year 2 evaluation included assessments of neuro-development (Bayley- 3 cognitive subscale), somatic growth, use of oxygen and respiratory medications, and the number and duration of rehospitalizations. NDI was defined as a cognitive score of $<85$, non-ambulant cerebral palsy, blindness or deafness.

Results: Of 800 babies entered into the trial, 696 were alive at 36 weeks PMA; 661 (95.0\%) were seen at 1 year and $630(90.5 \%)$ at 2 years. No significant differences were found across all assessed variables. Compared to placebo, $67.2 \%$ of infants receiving iNO survived free of NDI (versus $72.4 \%$; RR: 0.93 (95\%CI: $0.84,1.02)$ ) and $14.4 \%$ were prescribed inhaled steroids compared to $18.8 \%$ (RR: 0.76 (95\%CI: $0.54-1.09))$.

Conclusions: Inhaled NO at $5 \mathrm{ppm}$ for 7-21 days did not affect the overall rate of neurologic, developmental or respiratory disability at 2 years. Follow up at school age is ongoing. 